\title{
Methotrexate in Atherogenesis and Cholesterol Metabolism
}

\author{
Eric Coomes, ${ }^{1}$ Edwin S. L. Chan, ${ }^{1}$ and Allison B. Reiss ${ }^{2}$ \\ ${ }^{1}$ Department of Medicine, Division of Translational Medicine, New York University School of Medicine, NBV 16N1, 550 First Avenue, \\ New York, NY 10016, USA \\ ${ }^{2}$ Inflammation Section, Winthrop Research Institute, Department of Medicine, Winthrop University Hospital, 222 Station Plaza North, \\ Suite 502, Mineola, NY 11501-3893, USA
}

Correspondence should be addressed to Allison B. Reiss, areiss@winthrop.org

Received 15 October 2010; Revised 11 January 2011; Accepted 11 January 2011

Academic Editor: M. Jauhiainen

Copyright (C) 2011 Eric Coomes et al. This is an open access article distributed under the Creative Commons Attribution License, which permits unrestricted use, distribution, and reproduction in any medium, provided the original work is properly cited.

\begin{abstract}
Methotrexate is a disease-modifying antirheumatic drug commonly used to treat inflammatory conditions such as rheumatoid arthritis which itself is linked to increased cardiovascular risk. Treatments that target inflammation may also impact the cardiovascular system. While methotrexate improves cardiovascular risk, inhibition of the cyclooxygenase (COX)-2 enzyme promotes atherosclerosis. These opposing cardiovascular influences may arise from differing effects on the expression of proteins involved in cholesterol homeostasis. These proteins, ATP-binding cassette transporter (ABC) A1 and cholesterol 27-hydroxylase, facilitate cellular cholesterol efflux and defend against cholesterol overload. Methotrexate upregulates expression of cholesterol 27 hydroxylase and ABCA1 via adenosine release, while COX-2 inhibition downregulates these proteins. Adenosine, acting through the $\mathrm{A}_{2 A}$ and $\mathrm{A}_{3}$ receptors, may upregulate proteins involved in reverse cholesterol transport by cAMP-PKA-CREB activation and STAT inhibition, respectively. Elucidating underlying cardiovascular mechanisms of these drugs provides a framework for developing novel cardioprotective anti-inflammatory medications, such as selective $\mathrm{A}_{2 \mathrm{~A}}$ receptor agonists.
\end{abstract}

\section{Introduction}

Rheumatoid arthritis is a multisystem autoimmune disorder [1]. Inflammatory processes in rheumatoid arthritis lead to accelerated atherosclerosis and afflicted patients have an elevated risk for cardiovascular events leading to a higher mortality [1]. In particular, elevated levels of immune complexes and cytokines (tumor necrosis factors, interleukins, and interferons (IFN)) contribute to the pathogenesis of both the articular [2] and cardiovascular manifestations of rheumatoid arthritis [3]. As such, the cardiovascular actions of antiarthritic medications are an important determinant for multiple aspects of patient care [3].

Although traditional nonsteroidal anti-inflammatory drugs (NSAIDs) [4], cyclooxygenase (COX)-2 inhibitors [5], methotrexate [6], and other disease-modifying antirheumatic drugs (DMARDs) are effective treatments for pain and inflammation, each of these drugs is linked with particular adverse effects which necessitate consideration. While NSAIDs are associated with gastrointestinal complications [7], some COX-2 inhibitors are associated with an elevated risk of cardiovascular events [5]. The COX-2 inhibitor cardiovascular risk has resulted in a market recall of several of these medications [8]. Although still associated with some adverse effects, methotrexate has proven to be one of the safest antirheumatic drugs [9].

In contrast to COX-2 inhibitors, methotrexate has demonstrated atheroprotective properties [10]. Recent studies have begun unravelling the molecular interactions underpinning the cardiovascular influence of COX-2 inhibition $[11,12]$ and methotrexate [13]. While these drugs have multiple cellular effects, cardiovascular modulation in part relies upon the regulation of reverse cholesterol transport $[13,14]$. This review aims to provide an overview of the mechanisms by which methotrexate interacts with cholesterol homeostasis to modulate atherogenesis in inflammatory disease.

\section{Mechanism of Action of Methotrexate}

Methotrexate is an anti-inflammatory medication commonly used in the treatment of rheumatoid arthritis and 
other inflammatory diseases such as psoriasis and inflammatory bowel disease [9]. The primary anti-inflammatory actions of methotrexate are attributable to adenosine release, triggered by methotrexate's polyglutamate metabolites [6].

Adenosine, a nucleoside produced by many cells and tissues in response to physical or metabolic stresses, is an endogenous anti-inflammatory mediator [15]. The physiological effects of adenosine are mediated by G-protein coupled 7-transmembrane receptors [16] that exist on almost all human cell types [15]. Adenosine receptors are divided into four classes, $A_{1}, A_{2 A}, A_{2 B}$, and $A_{3}$, based on the differential selectivity of adenosine analogues and molecular structure [15].

It has been noted that some of the anti-inflammatory effects of methotrexate may be reversed by inhibition of the $A_{2 A}$ receptor [17]. Further research suggests that stimulation of the $A_{2 A}$ receptor interferes with inflammatory processes such as the biosynthesis and release of proinflammatory cytokines, inhibits oxidative activity, prevents platelet aggregation, and reduces adhesion and degranulation of neutrophils [18]. Together these results demonstrate that the $\mathrm{A}_{2 \mathrm{~A}}$ receptor plays a significant role in the mediation of inflammation $[17,18]$.

Although adenosine has anti-inflammatory properties principally through its $A_{2 A}[17]$ and $A_{3}$ [19] receptors, it can also act in contradictory fashions via alternate receptors [20]. Ligation of adenosine to the $A_{1}$ receptor initiates inflammatory processes [20]. The adenosine receptors influence inflammatory processes by modulating cAMP signalling cascades [20]. In particular, the opposing effects of $A_{2 A}$ and $A_{1}$ activation are accounted for, respectively, by an elevation or reduction in intracellular cAMP [20]. Consequently, the overall effect of adenosine on inflammatory processes depends upon the relative temporal and spatial distribution of the various adenosine receptors, as well as the influence of other effectors in the inflammatory milieu [20].

\section{Atherosclerosis and Reverse Cholesterol Transport}

3.1. Atherosclerotic Development. Atherosclerosis, an underlying cause of myocardial infarction and stroke, is an intricate process that comprises elements of both inflammation and lipid accumulation, characterized by the thickening of arterial walls due to the development of a fibrous plaque [21]. When lipid load processing is inadequate in the monocyte infiltrated subendothelial intimia [22], unregulated cholesterol depositions in macrophages transform them into foam cells, marking the formation of fatty streaks [21]. Subsequent development forms a fibrous plaque narrowing the arterial lumen [21].

3.2. Reverse Cholesterol Transport. One of the initiating factors in the development of atherosclerosis is a deregulation of cholesterol homeostatic mechanisms [23]. The metabolism and removal of excess cholesterol is required to prevent foam cell accumulation [24]. Reverse cholesterol transport (RCT) is the process that transports such cholesterol from extrahepatic cells to the liver and intestine for excretion [25]. By preventing lipid accumulation via the control of cholesterol efflux, RCT pathways provide a defensive mechanism against proatherogenic cholesterol overload [25].

Extrahepatic cells eliminate intracellular cholesterol via multiple mechanisms of RCT, including adenosine triphosphate binding cassette transporter (ABC) A1 [26], ABCG1 [27], and cholesterol 27-hydroxylase (27-hydroxylase) [28] pathways. Cholesterol may be removed to HDL using ABCA1 [26] and ABCG1 [27] or processed within the cell by 27hydroxylase prior to passive diffusion [28].

\subsection{ABCA1: Adenosine Triphosphate-Binding Cassette Trans-} porter A1. Excess cholesterol within the cell may be actively transported outward to maintain cholesterol balance [25]. In particular, ABCA1 acts as a rate-controlling transporter in this cholesterol efflux process [26]. Interactions of apolipoproteins with cholesterol-loaded cells stimulate the ABCA1 mediated transport of cholesterol and phospholipids to extracellular apoA-I [26]. A series of subsequent modifications ultimately lead to hepatic uptake and biliary excretion of cholesterol [29].

Mutations in ABCA1 cause a severe HDL deficiency syndrome known as Tangier disease [30]. The reduction in apoA-I initiated efflux of cholesterol leads to the accumulation of lipid deposits in tissues throughout the body [30]. Consequently, patients with this disease have a marked increase in the risk of coronary artery disease [31].

Although ABCA1 is normally constitutively expressed, in grossly atherosclerotic tissue, ABCA1 protein is reduced despite upregulation in ABCA1 mRNA [32]. This suggests that in the progression of atherosclerosis, posttranscriptional processes prevent the formation of ABCA1 [32]. Lee-Rueckert et al. [33] have demonstrated that ABCA1 expression is significantly attenuated by acidification. Since atherosclerotic tissue is gradually acidified as the lesion advances, this acid-dependent downregulation may partially account for the observed decrease in ABCA1 [34]. Given the association between ABCA1 mutation and atherosclerosis [31] as well as the observance of ABCA1 downregulation in atherogenesis [32], support is given to the notion that the dysregulation of ABCA1 facilitates the development of atherosclerosis.

3.4. Cholesterol 27-Hydroxylase. Although ABC-dependent RCT is the primary mechanism of cholesterol removal [26], the 27-hydroxylase pathway provides an alternative to the apoA-I-dependent process [28]. The mitochondrial cytochrome P450 27-hydroxylase enzyme is involved in the first oxidation step of cholesterol in the "acidic" pathway for biosynthesis of bile acids [35]. 27-hydroxylase is expressed at high levels in the THP-1 human monocytoid cell line, macrophages, and principal cell types in the atherogenic process such as human arterial endothelium and monocytes [35].

27-hydroxylase catalyzes the conversion of cholesterol to 27 -hydroxycholesterol and subsequently 3 - $\beta$-hydroxy-5cholestenoic acid [36]. Effectively, this enzyme converts 
cholesterol into an oxygenated derivative or oxysterol. Such oxysterols are generally present in trace quantities, but their levels rise within atheromas [37]. The rapid excretion and degradation of these oxysterols is facilitated by their ability to pass lipophilic membranes $[12,38]$.

In atheroma, the dominant oxysterol is 27-hydroxycholesterol, at levels up to one-hundred times greater than in circulation $[37,39]$. Given the faltering ability of ABCA1 to initiate RCT in advanced atheromas [32], the 27-hydroxylase mediated efflux pathway represents a major remaining defensive mechanism against excess cholesterol loading [38].

3.5. ABCA1 and 27-Hydroxylase Expression. At the level of gene expression, an interconnecting factor exists between ABCA1 and 27-hydroxylase [40, 41]. Oxysterols, including 27-hydroxycholesterol, bind and activate the liver $\mathrm{X}$ receptors (LXR) [40]. This interaction induces the nuclear translocation of an LXR-retinoid X receptor heterodimer, a transcription factor, which promotes the expression [42] of cholesterol metabolism genes, particularly ABCA1 [41].

\section{Immunological and Inflammatory Processes in Atherogenesis}

Accumulating evidence indicates that the systemic inflammatory load in lupus disrupts cholesterol dynamics, increasing vulnerability to cholesterol accumulation in cells of the artery wall, including macrophages and endothelium [43]. Patients with lupus [44] and rheumatoid arthritis [1] are known to have an increased risk for atheromatous cardiovascular disease. The immune-complex dysregulation seen in these conditions may play a role in the atherogenic process, a notion supported by evidence from cholesterol loading and efflux $[44,45]$.

In some inflammatory disease, antibodies to oxidized LDL are generated [45]. The resulting formation of oxLDL-antibody complexes enhances uptake of oxLDL into macrophages and promotes foam cell formation [44, 45]. Furthermore, stimulation of cultured human monocytes and THP-1 human monocytoid cells with the immune reactants IFN- $\gamma$ or immune complexes impede cellular cholesterol efflux by markedly decreasing 27-hydroxylase and ABCA1 [46]. Collectively, these immune and inflammatory mediators promote atherosclerosis by disabling mechanisms that prevent the cells of the artery wall from being overloaded with cholesterol, leading to the formation of lipid laden foam cells $[46,47]$.

Research suggests that IFN- $\gamma$ inhibits RCT by modifying signal transducer and activator of transcription (STAT) protein activity via two alternate mechanisms $[48,49]$. In the first process, IFN- $\gamma$ binds to the IFN receptor, initiating the dimerization of the two IFN- $\gamma$ receptor sub-units which phosphorylates the bound janus kinases $[48,50]$. The activated kinase phosphorylates the IFN- $\gamma$ receptor, recruiting STAT proteins [50]. These STAT proteins are tyrosine phosphorylated, dimerize and translocate to the nucleus, where they downregulate $\operatorname{LXR} \alpha$, which in turn decreases ABCA1 expression [48].
In the second process, IFN- $\gamma$ induces a calcium flux which activates the calcium/calmodulin-dependent protein kinase II (CaMKII) [49]. CaMKII directs the phosphorylation of the STAT residue, $\operatorname{Ser}^{727}$ which maximizes STAT activity [51]. The ability of both immune complexes and IFN- $\gamma$ to influence RCT and macrophage to foam cell conversion emphasizes the importance of cardiovascular considerations in the treatment of inflammatory autoimmune disorders [47].

\section{Mechanisms for Methotrexate Influence on Reverse Cholesterol Transport and Atherosclerosis}

Across a multiplicity of reviewed studies, COX-2 inhibition elevated cardiovascular risk [5] while methotrexate significantly reduces cardiovascular disease mortality amongst rheumatoid arthritis patients [10], despite some disagreement for patients with prior atherosclerotic development [52]. Given the fundamental role of ABCA1 and 27hydroxylase in regulating foam cell formation, we postulated a potential role for RCT in the atherosclerotic effects following treatment with COX-2 inhibitors and methotrexate [13]. The application of COX-2 inhibitors to THP-1 monocytes cultures resulted in significant decreases in the gene expression of 27-hydroxylase and ABCA1 [14]. The resultant reduction in ABCA1 and 27-hydroxylase protein creates an environment where cholesterol efflux is compromised, promoting foam cell formation [14].

Significantly, the effects of COX-2 inhibition on cholesterol metabolism and efflux may be reversed by the addition of adenosine $A_{2 A}$ receptor agonists [11]. Given the previously described ability of methotrexate to induce adenosine release [6], it is plausible that methotrexate is capable of upregulating RCT as its mechanism for atheroprotection.

Using RT-PCR and immunoblotting, the levels of expression of ABCA1 and 27-hydroxylase were evaluated in cells in the presence of COX-2 inhibitors or IFN- $\gamma$ with or without methotrexate [13]. Methotrexate reversed foam cell formation and the down regulation of ABCA1 and 27-hydroxylase induced by COX-2 inhibition or IFN- $\gamma$ [13]. However, this methotrexate-induced upregulation was prevented by an inhibition of adenosine $\mathrm{A}_{2 \mathrm{~A}}$ receptors [13]. Thus, the capacity of methotrexate to reduce the onset of atherosclerosis may be partially attributed to the adenosinedriven upregulation of ABCA1 and 27-hydroxylase which expedites cholesterol efflux [13].

The specific mechanism by which adenosine $A_{2 A}$ receptor ligation modifies 27-hydroxylase and ABCA1 expression has not yet been elucidated [12]. However, the inhibition of PKA prevents adenosine $\mathrm{A}_{2 A}$ stimulation of RCT proteins, demonstrating a dependency upon a cAMP-PKA-mediated process [46]. Generally, $\mathrm{A}_{2 A}$ receptor ligation activates adenylate cyclase, leading to the accumulation of cAMP [53]. cAMP activates PKA which subsequently activates CREB proteins [46]. These proteins then translocate into the nucleus, where it interacts with a consensus element in 


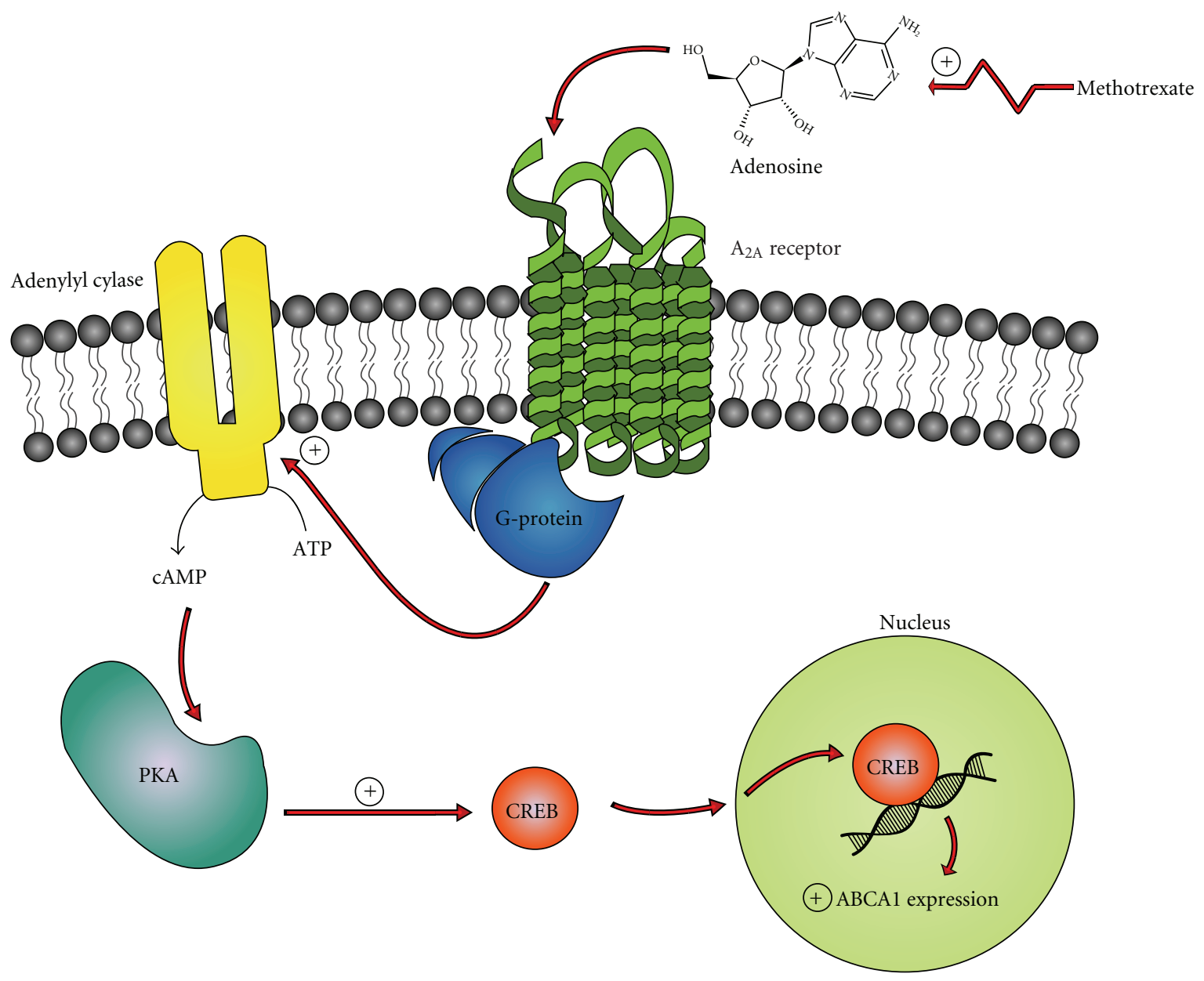

FIGURE 1: Regulation of adenosine triphosphate binding cassette transporter A1 (ABCA1) expression by adenosine. Methotrexate via numerous steps (jagged arrow) results in increased levels of adenosine. Adenosine activates the G-protein coupled receptor, $\mathrm{A}_{2 A}$, inducing an increase in adenylyl cyclase activity. The subsequent rise in cyclic adenosine monophosphate (cAMP) activates protein kinase A (PKA) which phosphorylates the cAMP response element-binding protein (CREB). CREB then translocates into the nucleus where it upregulates ABCA1 gene expression.

the gene promoter, modulating the expression of the target gene [46] (Figure 1).

As previously discussed, 27-hydroxycholesterol upregulates $\mathrm{ABCA1}$ expression $[40,41]$. A reasonable hypothesis is that $A B C A 1$ upregulation is dependent upon the increased expression of 27-hydoxylase. However, inhibition of the 27hydroxylase enzyme does not hinder the ability of adenosine $A_{2 A}$ ligation to upregulate $A B C A 1$, suggesting that the methotrexate-induced increase in ABCA1 expression is not contingent on this feedback loop [46].

In addition to stimulating increased ABCA1 expression, $\mathrm{A}_{2 A}$ ligation augments $\mathrm{ABCA} 1$ activity [54]. The generation of cAMP by $\mathrm{A}_{2 A}$ stimulation activates protein kinases and Epac [54], a guanine nucleotide exchange factor [55]. Protein kinase generates phospho-ABCA1 and Epac activates ABCA1 function [54]. Thus, methotrexate promotes cholesterol efflux and hinders atherogenesis by both upregulating and activating RCT proteins.

Beyond the $\mathrm{A}_{2 A}$ receptor, adenosine may counteract the effects of IFN- $\gamma$ via the $A_{3}$ receptor [49]. The addition of adenosine to IFN- $y$ stimulated cells decreases $\operatorname{ser}^{727}$ phosphorylation, thereby reducing STAT activity [49]. This decrease in STAT activity decreases the expression of genes involved in inflammation and lipid uptake [48]. Inhibition of the $A_{3}$ receptor reverses the suppressive effects of adenosine on STAT $\operatorname{ser}^{727}$ phosphorylation [49]. Given the role of STAT in suppressing RCT [48], this activity may additionally contribute to the antiatherosclerotic actions of methotrexate [49]. Although the $A_{3}$-mediated pathway has yet to be uncovered, it has been demonstrated that $\mathrm{A}_{3}$ receptor activation prevents the accumulation of $\mathrm{Ca}^{2+}[56]$. Thus, it is possible that $\mathrm{A}_{3}$ activity prevents the activation of CaMKII, which subsequently reduces the phosphorylation of $\operatorname{ser}^{727}$ [49] (Figure 2).

\section{Conclusion}

While methotrexate improves cardiovascular risk in a number of inflammatory diseases [10], COX-2 inhibition 


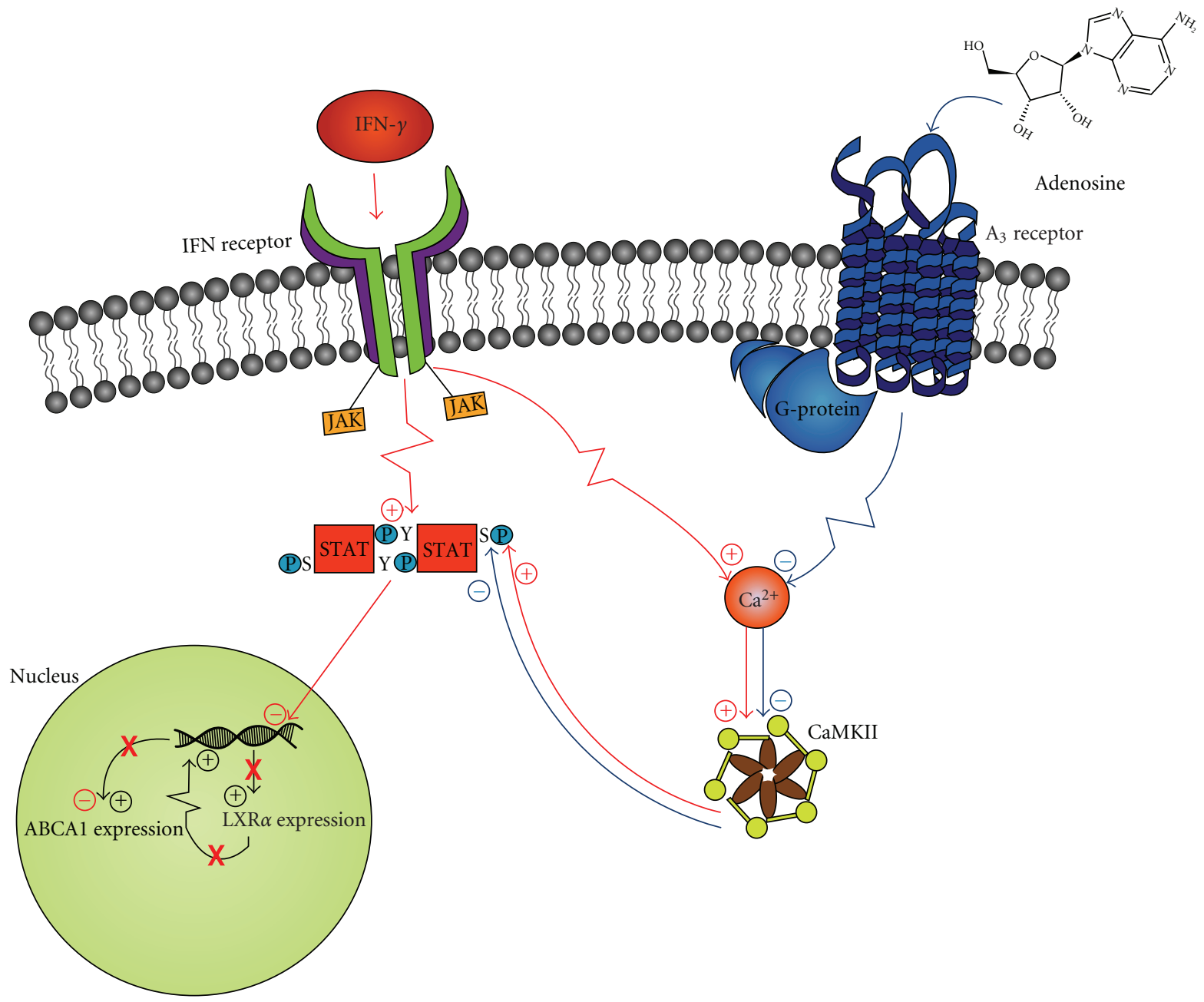

FIGURE 2: Abrogation of interferon (IFN)- $\gamma$ mediated downregulation of adenosine triphosphate binding cassette transporter A1 (ABCA1) by adenosine $\mathrm{A}_{3}$ receptor ligation. IFN- $\gamma$ binds the IFN receptor and subsequently decreases ABCA1 expression by increasing signal transducer and activator of transcription (STAT) protein activity via tyrosine (Y) and serine (S) phosphorylation. The IFN receptor induces a januskinase mediated process which tyrosine phosphorylates STAT. The serine is phosphorylated by a calcium/calmodulin-dependent protein kinase II (CaMKII) activated by a calcium flux initiated by the IFN receptor. These activated STAT proteins dimerize and translocate to the nucleus, where they downregulate liver X receptor (LXR) $\alpha$. While LXR normally upregulates ABCA1 expression, a decrease in LXR results in a consequent decrease in ABCA1 expression. Significantly, adenosine may counteract the effects of IFN- $\gamma$ on ABCA1 via the $A_{3}$ receptor. $\mathrm{A}_{3}$ activation prevents the accumulation of $\mathrm{Ca}^{2+}$ and may thus prevent the activation of CaMKII. This reduced activity will decrease the phosphorylation of $\mathrm{S}^{727}$ on STAT, decreasing STAT activity and thus preventing the downregulation of ABCA1.

promotes atherosclerosis [5]. These opposing cardiovascular influences of methotrexate and COX-2 inhibitors may arise from differing effects on the expression of ABCA1 and 27hydroxylase, key proteins in RCT [25]. While methotrexate upregulates their expression [13], COX-2 inhibition causes a downregulation [14]. Methotrexate increases the expression of these proteins via adenosine acting upon the $A_{2 A}[13]$ and $A_{3}$ receptors [49]. Activation of $A_{2 A}$ increases expression of the proteins and activity of ABCA1 by a cAMP-mediated process [46] while the $\mathrm{A}_{3}$ receptor counteracts the IFN$\gamma$ induced downregulation by inhibiting STAT [49]. Furthermore, as proposed in the Cardiovascular Inflammation Reduction Trial, methotrexate administration may provide additional antiatherosclerotic benefits in patients already receiving traditional cholesterol-lowering therapy, regardless of inflammatory disease status [57]. Thus, the understanding of the mechanisms by which these medications act to induce cardiovascular effects provides a platform for the design of novel and safer anti-inflammatory and antiatherosclerotic drugs in the future, such as selective adenosine $A_{2 A}$ receptor agonists.

\section{Declaration}

E.S.L. Chan declares that he holds a patent pertinent to King Pharmaceuticals on the use of adenosine $\mathrm{A}_{2 A}$ receptor antagonists to inhibit fibrosis. 


\section{Abbreviations}

IFN: Interferon

NSAID: Nonsteroidal anti-inflammatory drug

COX: Cyclooxygenase

DMARD: Disease modifying antirheumatic drug

cAMP: Cyclic adenosine monophosphate

RCT: $\quad$ Reverse cholesterol transport

ABC: $\quad$ Adenosine triphosphate binding cassette transporter

HDL: High density lipoprotein

apoA-I: Apolipoprotein AI

mRNA: Messenger ribonucleic acid

THP-1: Human acute monocytic leukemia cell line

LXR: Liver $\mathrm{X}$ receptor

ox-LDL: Oxidized low density lipoprotein

STAT: $\quad$ Signal transducer and activator of transcription

PKA: Calcium/calmodulin-dependent protein kinase II, protein kinase A

CREB: cAMP response element-binding

ATP: $\quad$ Adenosine triphosphate

Epac: Exchange protein activated by cAMP.

\section{Acknowledgments}

This work was supported by Grants from the U.S. National Institutes of Health (AR057544), the Scleroderma Foundation, the Arthritis Foundation, and the NYU-HHC Clinical and Translational Science Institute (UL1RR029893) (E.S.L.C.). Additionally, this work was supported in part by an Innovative Research Grant from the Arthritis Foundation National Center (A.B.R.). The authors would also like to thank Farheen Manji, at McMaster University in Hamilton, $\mathrm{ON}$, for her assistance with the figures.

\section{References}

[1] C. Meune, E. Touzé, L. Trinquart, and Y. Allanore, "High risk of clinical cardiovascular events in rheumatoid arthritis: levels of associations of myocardial infarction and stroke through a systematic review and meta-analysis," Archives of Cardiovascular Diseases, vol. 103, no. 4, pp. 253-261, 2010.

[2] M. Feldmann, F. M. Brennan, and R. N. Maini, "Role of cytokines in rheumatoid arthritis," Annual Review of Immunology, vol. 14, pp. 397-440, 1996.

[3] C. Tanasescu, C. Jurcut, R. Jurcut, and C. Ginghina, "Vascular disease in rheumatoid arthritis: from subclinical lesions to cardiovascular risk," European Journal of Internal Medicine, vol. 20, no. 4, pp. 348-354, 2009.

[4] D. E. Furst, R. W. Ulrich, and C. Varkey-Altamirano, "Nonsteroidal anti-inflammatory drugs, disease modifying antirheumatic drugs, nonopiod analgesics \& drugs used in gout," in Basic and Clinical Pharmacology, B. G. Katzung, S. B. Masters, and A. J. Trevor, Eds., pp. 621-642, McGraw Hill, New York, NY, USA, 11th edition, 2009.

[5] G. A. FitzGerald, "Coxibs and cardiovascular disease," New England Journal of Medicine, vol. 351, no. 17, pp. 1709-1711, 2004.
[6] E. S. L. Chan and B. N. Cronstein, "Methotrexatehow does it really work?" Nature Reviews Rheumatology, vol. 6, no. 3, pp. 175-178, 2010.

[7] J. Castellsague, C. N. Holick, C. C. Hoffman, V. Gimeno, M. R. Stang, and S. Perez-Gutthann, "Risk of upper gastrointestinal complications associated with cyclooxygenase- 2 selective and nonselective nonsteroidal antiinflammatory drugs," Pharmacotherapy, vol. 29, no. 12, pp. 1397-1407, 2009.

[8] S. X. Sun, K. Y. Lee, C. T. Bertram, and J. L. Goldstein, "Withdrawal of COX-2 selective inhibitors rofecoxib and valdecoxib: impact on NSAID and gastroprotective drug prescribing and utilization," Current Medical Research and Opinion, vol. 23, no. 8, pp. 1859-1866, 2007.

[9] E. S. L. Chan, S. N. Oliver, and B. N. Cronstein, "Immunomodulating pharmaceuticals," in Clinical Immunology: Principles and Practice, R. R. Rich, T. A. Fleisher, W. T. Shearer, H. W. Schroeder, A. J. Frew, and C. M. Weyand, Eds., pp. 90-1-90-9, Mosby Elsevier, Philadelphia, Pa, USA, 3rd edition, 2008.

[10] S. L. Westlake, A. N. Colebatch, J. Baird et al., "The effect of methotrexate on cardiovascular disease in patients with rheumatoid arthritis: a systematic literature review," Rheumatology, vol. 49, no. 2, pp. 295-307, 2009.

[11] A. Reiss, H. Zhang, S. Edelman et al., "Unraveling coxib atherogenicity: adenosine A2A receptor agonists reverse disruption of cholesterol efflux induced by cyclooxygenase (COX)-2 inhibition," Inflammation Research, vol. 55, pp. S117-S118, 2006.

[12] A. B. Reiss, F. Anwar, E. S. L. Chan, and K. Anwar, "Disruption of cholesterol efflux by coxib medications and inflammatory processes: link to increased cardiovascular risk," Journal of Investigative Medicine, vol. 57, no. 6, pp. 695-702, 2009.

[13] A. B. Reiss, S. E. Carsons, K. Anwar et al., "Atheroprotective effects of methotrexate on reverse cholesterol transport proteins and foam cell transformation in human THP-1 monocyte/macrophages," Arthritis and Rheumatism, vol. 58, no. 12, pp. 3675-3683, 2008.

[14] E. S. L. Chan, H. Zhang, P. Fernandez et al., "Effect of cyclooxygenase inhibition on cholesterol efflux proteins and atheromatous foam cell transformation in THP-1 human macrophages: a possible mechanism for increased cardiovascular risk," Arthritis Research and Therapy, vol. 9, Article ID R4, 2007.

[15] V. Ralevic and G. Burnstock, "Receptors for purines and pyrimidines," Pharmacological Reviews, vol. 50, no. 3, pp. 413492, 1998.

[16] M. E. Olah and G. L. Stiles, "The role of receptor structure in determining adenosine receptor activity," Pharmacology and Therapeutics, vol. 85, no. 2, pp. 55-75, 2000.

[17] M. C. Montesinos, A. Desai, and B. N. Cronstein, "Suppression of inflammation by low-dose methotrexate is mediated by adenosine A receptor but not A receptor activation in thioglycollate-induced peritonitis," Arthritis Research and Therapy, vol. 8, no. 2, Article ID R53, 2006.

[18] J. A. McPherson, K. G. Barringhaus, G. G. Bishop et al., "Adenosine A receptor stimulation reduces inflammation and neointimal growth in a murine carotid ligation model," Arteriosclerosis, Thrombosis, and Vascular Biology, vol. 21, no. 5, pp. 791-796, 2001.

[19] P. A. Borea, S. Gessi, S. Bar-Yehuda, and P. Fishman, "A adenosine receptor: pharmacology and role in disease," Handbook of Experimental Pharmacology, vol. 193, pp. 297-327, 2009. 
[20] S. Nakav, C. Chaimovitz, Y. Sufaro et al., "Anti-inflammatory preconditioning by agonists of adenosine A1 receptor," PLoS One, vol. 3, no. 5, Article ID e2107, 2008.

[21] R. Ross, "Atherosclerosis-an inflammatory disease," New England Journal of Medicine, vol. 340, no. 2, pp. 115-126, 1999.

[22] P. Libby, "Changing concepts of atherogenesis," Journal of Internal Medicine, vol. 247, no. 3, pp. 349-358, 2000.

[23] A. Von Eckardstein, J. R. Nofer, and G. Assmann, "High density lipoproteins and arteriosclerosis role of cholesterol efflux and reverse cholesterol transport," Arteriosclerosis, Thrombosis, and Vascular Biology, vol. 21, no. 1, pp. 13-27, 2001.

[24] H. S. Kruth, "Macrophage foam cells and atherosclerosis," Frontiers in Bioscience, vol. 6, pp. D429-D455, 2001.

[25] R. Ohashi, H. Mu, X. Wang, Q. Yao, and C. Chen, "Reverse cholesterol transport and cholesterol efflux in atherosclerosis," Monthly Journal of the Association of Physicians, vol. 98, no. 12, pp. 845-856, 2005.

[26] J. F. Oram and R. M. Lawn, "ABCA1: the gatekeeper for eliminating excess tissue cholesterol," Journal of Lipid Research, vol. 42, no. 8, pp. 1173-1179, 2001.

[27] N. Terasaka, N. Wang, L. Yvan-Charvet, and A. R. Tall, "Highdensity lipoprotein protects macrophages from oxidized lowdensity lipoprotein-induced apoptosis by promoting efflux of 7-ketocholesterol via ABCG1," Proceedings of the National Academy of Sciences of the United States of America, vol. 104, no. 38, pp. 15093-15098, 2007.

[28] A. Babiker, O. Andersson, E. Lund et al., "Elimination of cholesterol in macrophages and endothelial cells by the sterol 27-hydroxylase mechanism. Comparison with high density lipoprotein- mediated reverse cholesterol transport," Journal of Biological Chemistry, vol. 272, no. 42, pp. 26253-26261, 1997.

[29] X. Wang and D. J. Rader, "Molecular regulation of macrophage reverse cholesterol transport," Current Opinion in Cardiology, vol. 22, no. 4, pp. 368-372, 2007.

[30] G. D. Kolovou, D. P. Mikhailidis, K. K. Anagnostopoulou, S. S. Daskalopoulou, and D. V. Cokkinos, "Tangier disease four decades of research: a reflection of the importance of HDL," Current Medicinal Chemistry, vol. 13, no. 7, pp. 771-782, 2006.

[31] I. Iatan, K. Alrasadi, I. Ruel, K. Alwaili, and J. Genest, "Effect of ABCA1 mutations on risk for myocardial infarction," Current Atherosclerosis Reports, vol. 10, no. 5, pp. 413-426, 2008.

[32] C. Albrecht, S. Soumian, J. S. Amey et al., "ABCA1 expression in carotid atherosclerotic plaques," Stroke, vol. 35, no. 12, pp. 2801-2806, 2004.

[33] M. Lee-Rueckert, J. Lappalainen, H. Leinonen, T. Pihlajamaa, M. Jauhiainen, and P. T. Kovanen, "Acidic extracellular environments strongly impair ABCA1-mediated cholesterol efflux from human macrophage foam cells," Arteriosclerosis, Thrombosis, and Vascular Biology, vol. 30, no. 9, pp. 1766$1772,2010$.

[34] M. Naghavi, R. John, S. Naguib et al., " $\mathrm{pH}$ heterogeneity of human and rabbit atherosclerotic plaques; a new insight into detection of vulnerable plaque," Atherosclerosis, vol. 164, no. 1, pp. 27-35, 2002.

[35] A. B. Reiss and A. D. Glass, "Atherosclerosis: immune and inflammatory aspects," Journal of Investigative Medicine, vol. 54, no. 3, pp. 123-131, 2006.

[36] V. M. Olkkonen and M. Lehto, "Oxysterols and oxysterol binding proteins: role in lipid metabolism and atherosclerosis," Annals of Medicine, vol. 36, no. 8, pp. 562-572, 2004.

[37] I. Björkhem and U. Diczfalusy, "Oxysterols: friends, foes, or just fellow passengers?" Arteriosclerosis, Thrombosis, and Vascular Biology, vol. 22, no. 5, pp. 734-742, 2002.
[38] I. Bjorkhem, O. Andersson, U. Diczfalusy et al., "Atherosclerosis and sterol 27-hydroxylase: evidence for a role of this enzyme in elimination of cholesterol from human macrophages," Proceedings of the National Academy of Sciences of the United States of America, vol. 91, no. 18, pp. 8592-8596, 1994.

[39] A. Babiker, S. Dzeletovic, B. Wiklund et al., "Patients with atherosclerosis may have increased circulating levels of 27hydroxycholesterol and cholestenoic acid," Scandinavian Journal of Clinical and Laboratory Investigation, vol. 65, no. 5, pp. 365-376, 2005.

[40] X. Fu, J. G. Menke, Y. Chen et al., "27-hydroxycholesterol is an endogenous ligand for liver $\mathrm{X}$ receptor in cholesterol-loaded cells," Journal of Biological Chemistry, vol. 276, no. 42, pp. 38378-38387, 2001.

[41] S. Murthy, E. Born, S. N. Mathur, and F. J. Field, "LXR/RXR activation enhances basolateral efflux of cholesterol in CaCo-2 cells," Journal of Lipid Research, vol. 43, no. 7, pp. 1054-1064, 2002.

[42] P. A. Edwards, M. A. Kennedy, and P. A. Mak, "LXRs; Oxysterol-activated nuclear receptors that regulate genes controlling lipid homeostasis," Vascular Pharmacology, vol. 38, no. 4, pp. 249-256, 2002.

[43] A. B. Reiss, "Effects of inflammation on cholesterol metabolism: impact on systemic lupus erythematosus," Current Rheumatology Reports, vol. 11, no. 4, pp. 255-260, 2009.

[44] J. George, D. Harats, B. Gilburd, Y. Levy, P. Langevitz, and Y. Shoenfeld, "Atherosclerosis-related markers in systemic lupus erythematosus patients: the role of humoral immunity in enhanced atherogenesis," Lupus, vol. 8, no. 3, pp. 220-226, 1999.

[45] G. Virella and M. F. Lopes-Virella, "Atherogenesis and the humoral immune response to modified lipoproteins," Atherosclerosis, vol. 200, no. 2, pp. 239-246, 2008.

[46] A. B. Reiss, M. M. Rahman, E. S. L. Chan, M. C. Montesinos, N. W. Awadallah, and B. N. Cronstein, "Adenosine A receptor occupancy stimulates expression of proteins involved in reverse cholesterol transport and inhibits foam cell formation in macrophages," Journal of Leukocyte Biology, vol. 76, no. 3, pp. 727-734, 2004.

[47] A. B. Reiss, N. W. Awadallah, S. Malhotra et al., "Immune complexes and IFN- $\gamma$ decrease cholesterol 27-hydroxylase in human arterial endothelium and macrophages," Journal of Lipid Research, vol. 42, no. 11, pp. 1913-1922, 2001.

[48] X. R. Hao, D. L. Cao, Y. W. Hu et al., "IFN- $\gamma$ down-regulates ABCA1 expression by inhibiting LXR $\alpha$ in a JAK/STAT signaling pathway-dependent manner," Atherosclerosis, vol. 203, no. 2, pp. 417-428, 2009.

[49] K. E. Barnholt, R. S. Kota, H. H. Aung, and J. C. Rutledge, "Adenosine blocks IFN- $\gamma$-induced phosphorylation of STAT1 on serine 727 to reduce macrophage activation," Journal of Immunology, vol. 183, no. 10, pp. 6767-6777, 2009.

[50] J. E. McLaren and D. P. Ramji, "Interferon gamma: a master regulator of atherosclerosis," Cytokine and Growth Factor Reviews, vol. 20, no. 2, pp. 125-135, 2009.

[51] J. S. Nair, C. J. DaFonseca, A. Tjernberg et al., "Requirement of $\mathrm{Ca}^{2+}$ and CaMKII for Stat1 Ser-727 phosphorylation in response to IFN- $\gamma$," Proceedings of the National Academy of Sciences of the United States of America, vol. 99, no. 9, pp. 59715976, 2002.

[52] R. B. M. Landewé, B. E. E. M. Van Den Borne, F. C. Breedveld, and B. A. C. Dijkmans, "Methotrexate effects in patients 
with rheumatoid arthritis with cardiovascular comorbidity," Lancet, vol. 355, no. 9215, pp. 1616-1617, 2000.

[53] J. Lynge, G. Schulte, N. Nordsborg, B. B. Fredholm, and Y. Hellsten, "Adenosine A receptors modulate cAMP levels and induce CREB but not ERK1/2 and p38 phosphorylation in rat skeletal muscle cells," Biochemical and Biophysical Research Communications, vol. 307, no. 1, pp. 180-187, 2003.

[54] T. C. Bingham, E. A. Fisher, S. Parathath, A. B. Reiss, E. S. Chan, and B. N. Cronstein, "A2A adenosine receptor stimulation decreases foam cell formation by enhancing ABCA1-dependent cholesterol efflux," Journal of Leukocyte Biology, vol. 87, no. 4, pp. 683-690, 2010.

[55] J. L. Bos, "Epac: a new cAMP target and new avenues in cAMP research," Nature Reviews Molecular Cell Biology, vol. 4, no. 9, pp. 733-738, 2003.

[56] L. Martin, S. C. Pingle, D. M. Hallam, L. P. Rybak, and V. Ramkumar, "Activation of the adenosine A receptor in RAW 264.7 cells inhibits lipopolysaccharide-stimulated tumor necrosis factor- $\alpha$ release by reducing calcium-dependent activation of nuclear factor- $\kappa \mathrm{B}$ and extracellular signal-regulated kinase 1/2," Journal of Pharmacology and Experimental Therapeutics, vol. 316, no. 1, pp. 71-78, 2006.

[57] P. M. Ridker, "Testing the inflammatory hypothesis of atherothrombosis: scientific rationale for the cardiovascular inflammation reduction trial (CIRT)," Journal of Thrombosis and Haemostasis, vol. 7, no. 1, pp. 332-339, 2009. 


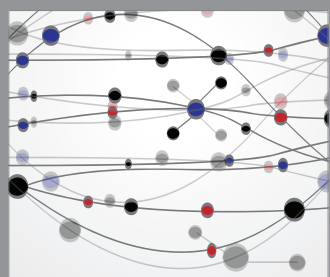

The Scientific World Journal
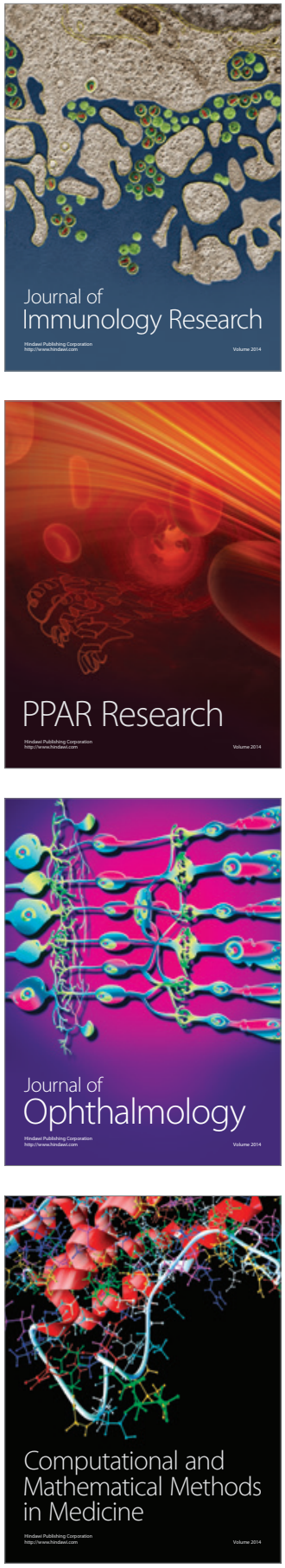

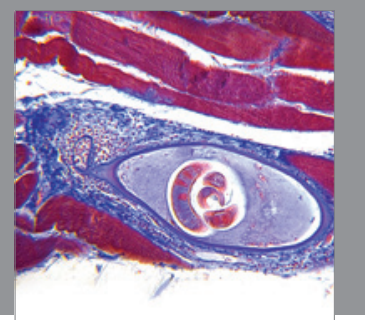

Gastroenterology

Research and Practice
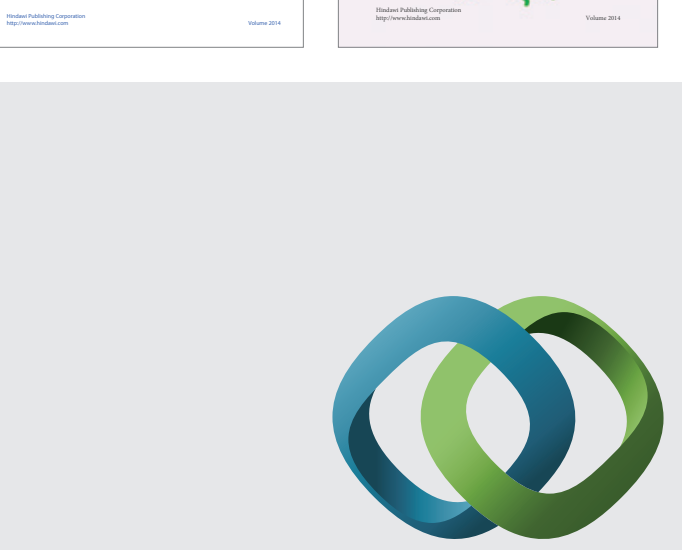

\section{Hindawi}

Submit your manuscripts at

http://www.hindawi.com
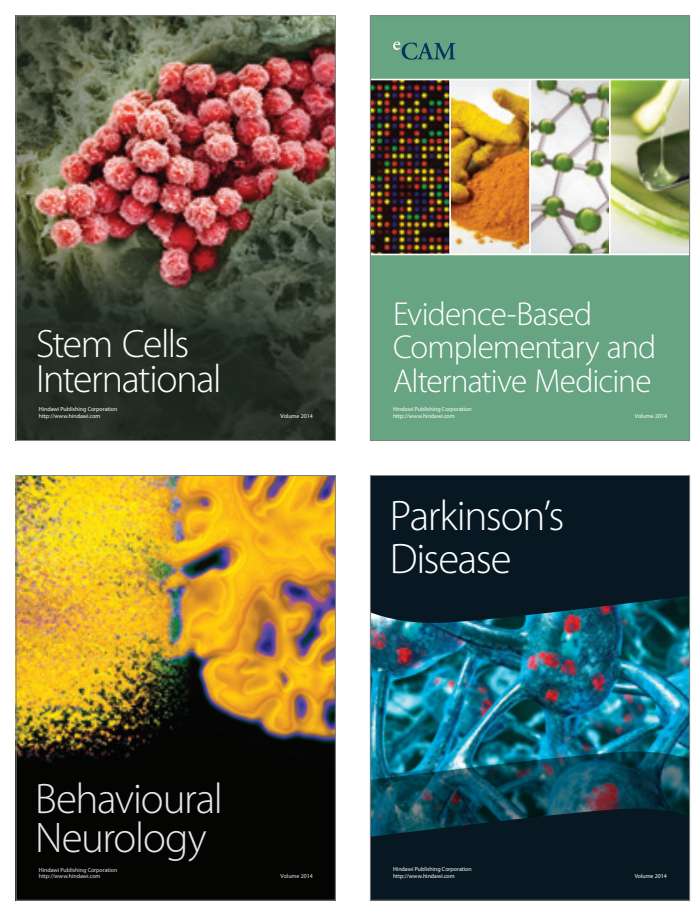

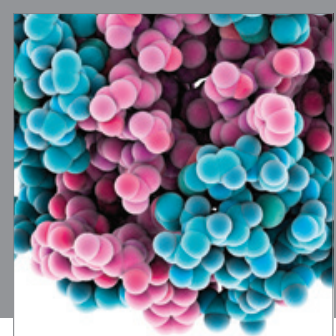

Journal of
Diabetes Research

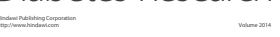

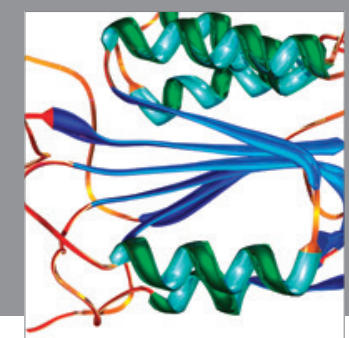

Disease Markers
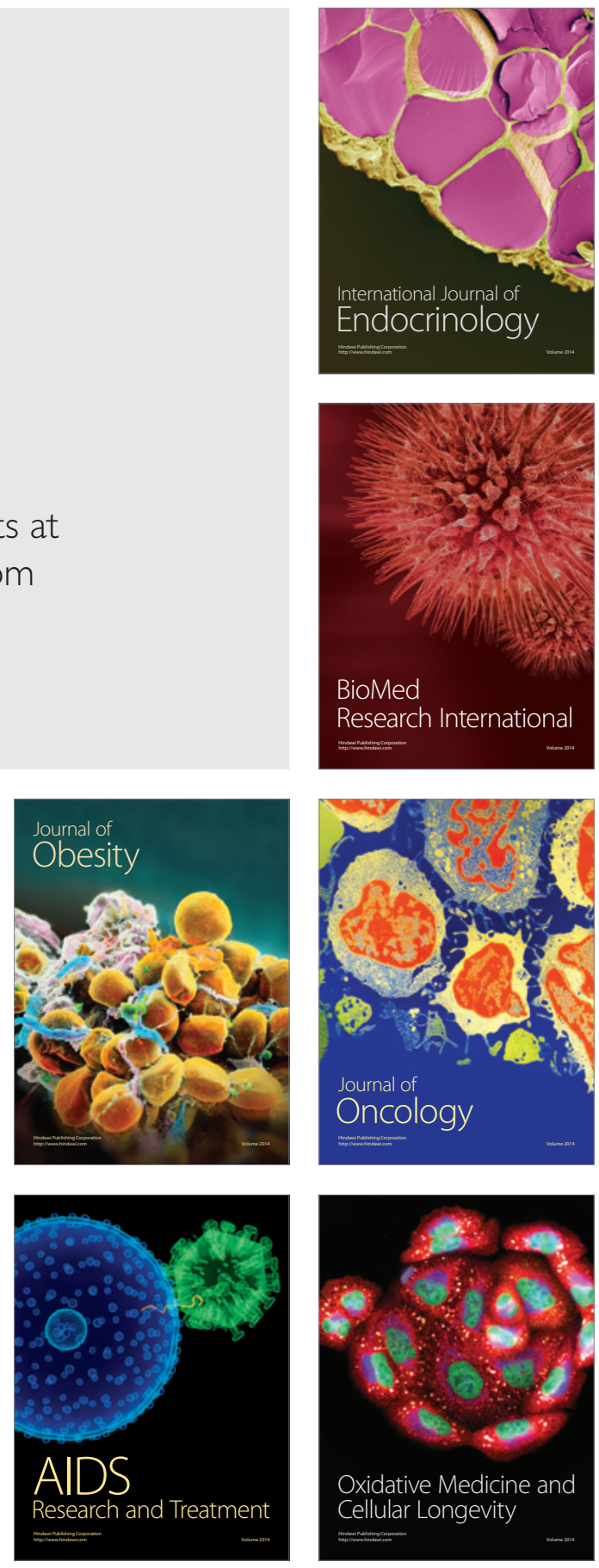